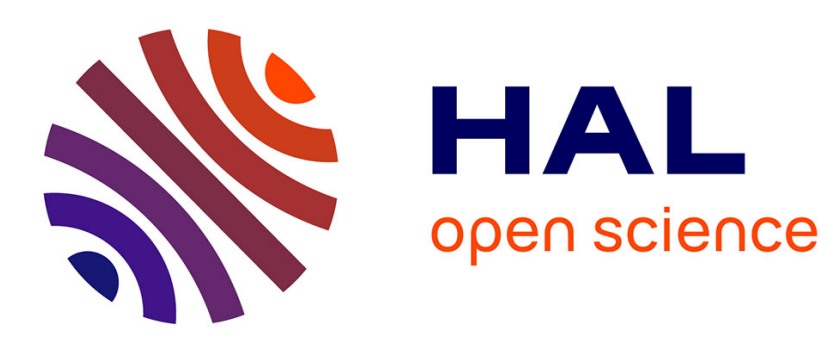

\title{
Perception of violin soundpost tightness through playing and listening tests
}

Lei Fu, Claudia Fritz, Gary Scavone

\section{To cite this version:}

Lei Fu, Claudia Fritz, Gary Scavone. Perception of violin soundpost tightness through playing and listening tests. Journal of the Acoustical Society of America, 2021, 150 (1), pp.540-550. 10.1121/10.0005587 . hal-03313013

\section{HAL Id: hal-03313013 https://hal.science/hal-03313013}

Submitted on 24 Nov 2021

HAL is a multi-disciplinary open access archive for the deposit and dissemination of scientific research documents, whether they are published or not. The documents may come from teaching and research institutions in France or abroad, or from public or private research centers.
L'archive ouverte pluridisciplinaire HAL, est destinée au dépôt et à la diffusion de documents scientifiques de niveau recherche, publiés ou non, émanant des établissements d'enseignement et de recherche français ou étrangers, des laboratoires publics ou privés. 


\section{Perception of violin soundpost tightness through playing and listening tests}

Lei Fu, Claudia Fritz, and Gary Scavone

Citation: The Journal of the Acoustical Society of America 150, 540 (2021); doi: 10.1121/10.0005587

View online: https://doi.org/10.1121/10.0005587

View Table of Contents: https://asa.scitation.org/toc/jas/150/1

Published by the Acoustical Society of America

\section{ARTICLES YOU MAY BE INTERESTED IN}

Automatic responses to musical intervals: Contrasts in acoustic roughness predict affective priming in Western listeners

The Journal of the Acoustical Society of America 150, 551 (2021); https://doi.org/10.1121/10.0005623

Reflections on "Parametric acoustic array," source of virtual-array sonars

The Journal of the Acoustical Society of America 150, R1 (2021); https://doi.org/10.1121/10.0005487

The bassoon tonehole lattice: Links between the open and closed holes and the radiated sound spectrum The Journal of the Acoustical Society of America 150, 398 (2021); https://doi.org/10.1121/10.0005627

Accelerating frequency-domain numerical methods for weakly nonlinear focused ultrasound using nested meshes

The Journal of the Acoustical Society of America 150, 441 (2021); https://doi.org/10.1121/10.0005655

Directional infrasound sensing using acoustic metamaterials

The Journal of the Acoustical Society of America 150, 367 (2021); https://doi.org/10.1121/10.0005594

Learning spectro-temporal representations of complex sounds with parameterized neural networks

The Journal of the Acoustical Society of America 150, 353 (2021); https://doi.org/10.1121/10.0005482

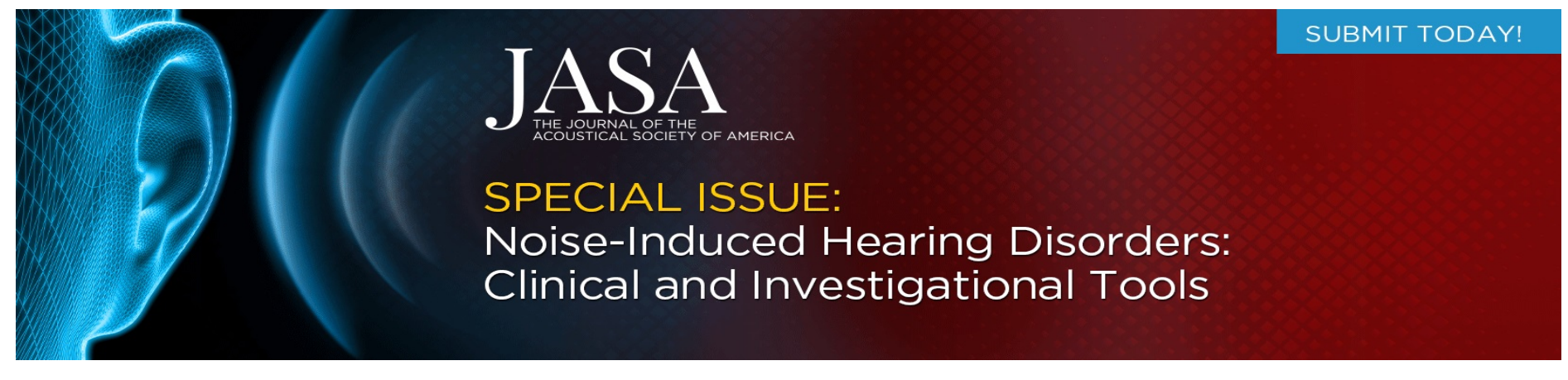




\title{
Perception of violin soundpost tightness through playing and listening tests
}

\author{
Lei Fu, ${ }^{1, a)}$ Claudia Fritz, ${ }^{2}$ and Gary Scavone ${ }^{3}$ \\ ${ }^{1}$ Department of Mechatronic Engineering, Luoyang Institute of Science and Technology, Luoyang, Henan 471023, China \\ ${ }^{2}$ Institut Jean le Rond d'Alembert, UMR 7190, CNRS/Sorbonne Université, Paris 75005, France \\ ${ }^{3}$ Computational Acoustic Modeling Laboratory, McGill University, Montreal, Quebec H3A IE3, Canada
}

\begin{abstract}
:
This study involved playing and listening (using recorded sounds) experiments to investigate how changes in soundpost length (for a fixed soundpost position) affect the perceptual qualities of the violin and what the threshold of change is below which players and luthiers do not perceive differences. A length-adjustable carbon fiber soundpost was employed. During the playing experiment, subjects played a provided violin on which the soundpost length was modified by the experimenter to find their optimal soundpost lengths. Then the experimenter varied the soundpost length randomly in ten trials within $\pm 0.11 \mathrm{~mm}$ around their optimal lengths and asked subjects to always compare with the previous setting. The results showed that subjects' optimal soundpost lengths varied from 0.132 to $0.616 \mathrm{~mm}$ relative to the original length $(53 \mathrm{~mm})$, but subjects could not recognize length variation of $0.11 \mathrm{~mm}$ or less at above chance levels. During the listening experiment, subjects listened to 16 pairs of recordings through a computer interface and were asked, for each pair, whether the violin setup was the same or different. The results showed that subjects could differentiate soundpost lengths with a difference of about $0.198 \mathrm{~mm}$ at better than chance level. @ 2021 Acoustical Society of America. https://doi.org/10.1121/10.0005587
\end{abstract}

(Received 3 December 2020; revised 18 May 2021; accepted 20 June 2021; published online 22 July 2021)

[Editor: Psyche Loui]

Pages: $540-550$

\section{INTRODUCTION}

The soundpost (SP) of a violin is an essential component of the instrument, providing structural support between the top and back plates and also a means of adjustment in the assembled instrument. According to luthiers, subtle changes to the SP dimensions or position can result in significant variations in the violin sound and playing qualities. The SP is typically made of the same wood as the top plate, and it is a cylinder of approximately $0.7 \mathrm{~g}, 6 \mathrm{~mm}$ diameter, and a bit longer than $50 \mathrm{~mm}$ (Bissinger, 1995). As stated by Savart (1840), the SP can help transmit the vibrations from the top plate to the back plate. Through experiments, he also proved that the first acoustical purpose of the SP is to introduce asymmetry to the violin.

Nadarajah (2018) summarized three factors that luthiers consider when setting up a SP and ordered them in terms of acoustical importance as "tightness," "position," and "fit." Makers all agree that the SP should be fit for full wood-towood contact with the plates, such that the post ends are uniformly in contact with the plates across their entire diameter. Also, everyone agrees that the SP must be tight enough to stay in place but not "too tight" (e.g., Johnson and Courtnall, 1999), even if this is hard to quantify, so as not to damage the plates. But all three aspects are co-dependent in several ways. For example, makers commonly adjust SP position to address perceived tone quality issues, but this

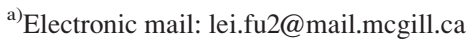

may also affect tightness and fit because the violin plates are generally not parallel. It is expected that varying any of these three factors will influence the coupling between the SP and the plates. That said, the process of adjusting the SP in response to player preferences remains largely based on empirical experience. This paper focuses on the perception of SP tightness variations while keeping position and fit constant.

Many researchers have studied the function of the SP through comparison between the violin with SP and without SP (no-SP). For instance, Jansson et al. (1970) employed hologram interferometry to study the resonances of the violin plates and fully assembled body. They found that a nodal line or a nodal area appeared on the interferograms of the plates around the position of the SP when the SP was in place and that the plate resonance frequencies increased with a SP compared to no-SP. Schelleng (1971) approximated the violin body as a closed cigar box and the SP as immovable to explain the effect of the SP in enhancing the sound radiation through top plate asymmetry. He also considered the case with a movable post to show that significant motion of the back plate can be attributed to the ribs, rather than the SP. Using a modal analysis method, Bissinger (1995) calculated the radiation efficiency of the violin. He observed a very considerable radiation efficiency enhancement of SP over no-SP in the region of $500-800 \mathrm{~Hz}$, in which there are some very important peaks in the response or radiativity curves. Overall, the average radiation efficiency increased by $17 \%$ with the SP installed. Saldner et al. 
(1996) studied the action of the SP by employing a TV-holography technique to visualize the modal patterns of an unvarnished violin in real time. Compared to the no-SP condition, they found that the main vibrations in the top plates are shifted to the opposite side of the SP, with only small vibrations or a nodal line at the SP position. In this way, the SP makes it possible for the symmetric vibration modes to be excited by the bridge. Jansson (2004) compared the violin with SP and without SP using measured bridge admittances. He found that the magnitude of the "bridge hill" is the highest with SP, while the magnitude of a peak at approximately $550 \mathrm{~Hz}$ is the highest without the SP.

More recently, scholars have employed computational violin models to study the influence of the SP on the violin. Nadarajah (2018) developed a simplified theoretical model of the violin and the SP, which he used to examine aspects of the setup (e.g., tightness, fit, and position). The results demonstrated that increasing the tightness of the SP led to an increase in the resonance frequencies of the model. The author concluded that the slightly off-centre position of the SP maximized the net volume change of the violin body. Gough $(2017,2018)$ studied the function of the SP using COMSOL shell structure finite element analysis computations. He found that both the SP and bassbar can break the symmetry of the empty violin body shell and introduce asymmetric coupled modes. Thus, between them, there is a symmetrybreaking competition. The SP and bassbar influence the tonal balance of the violin over the whole playing range and the intensity of the radiated sound.

Most of the previous studies on violin SPs focused on physical or acoustical aspects and were concerned with the role of the SP (installed vs removed) or general trends in its positioning. How the SP affects the perceptual qualities of the violin, however, has not been rigorously investigated, despite the fact that luthiers consider it to be an important means of setup adjustment. In designing a perceptual study to evaluate the influence of the SP, several practical constraints had to be addressed. First, it is not possible for a violin to be played under full tension without a SP, as it would likely be damaged. Second, it is extremely difficult to specify repeated position changes of a traditional SP with sufficient accuracy and speed during a playing experiment. Therefore, this study was designed to investigate correlations between a change in length (or tightness) of the SP and variations of the perceived quality of the violin through both playing and listening tests with skilled violinists and luthiers, using an easily adjustable carbon fiber SP.

This paper is divided in two main sections, Sec. II reporting the playing tests and Sec. III the listening tests. In each section, the methodology is presented first, followed by the results. The paper ends with a conclusion and a discussion of the results (Sec. IV).

\section{PLAYING EXPERIMENT}

Our initial interest for a perceptual study was to investigate changes in SP position. However, results of a pilot study (conducted at the 2018 Oberlin Violin Acoustics
Workshop organized by the Violin Society of America) demonstrated difficulties in accurate and reasonably fast repeated positioning at specific locations inside the violin soundbox, as well as the need for an experienced luthier to be present for the duration of the experiment. The availability of a commercial length-adjustable carbon fiber SP instead offered the ability to study the perception of SP length changes. With a bit of practice, it was found that the length adjustments could be accomplished by one of the experimenters within a minute or less, thus obviating the need (and cost) for a luthier to be present throughout the experiments.

\section{A. Materials and methods}

\section{General design}

This experiment explores how changes in SP length affect the perceptual qualities of the violin and whether there is a threshold of change below which players do not perceive differences. A violin installed with a lengthadjustable carbon fiber SP was employed. The experiment was designed as a sequence of playing tests. An experimenter was present to change the SP length. Violinists and luthiers were invited to participate. The experiment involved two phases. During the first phase, subjects played and described their feelings about the violin with different SP settings in order to find their optimal SP length. During the second phase, the experimenter randomly increased, decreased, or did not change the SP length in ten trials around their optimal length. For each trial, subjects were asked to play the violin, comparing it with the previous setting, and to decide whether they were the same or different.

Players were asked to use their own bows to play and evaluate the violin, as they typically use their own bows when testing violins in real life. Luthiers were given the option of either using their own bow or using a provided bow. This experiment took place in a room free of strong resonances and a relatively low reverberation time. The area of the experiment room was approximately $26.7 \mathrm{~m}^{2}$.

\section{Soundpost and violin}

A length-adjustable carbon fiber SP (Anima Nova, Rastatt, Germany) was employed for this study, as shown in Fig. 1. The upper cylinder shell of the SP possessing a scale on its bottom is sheathed with the lower cylinder through an internal thread, and one can increase or decrease the SP length by turning the upper cylinder shell anticlockwise or clockwise. A vertical line indicated on the surface of the lower cylinder acts as the pointer of the scale, which has five numbers from 0 to 4 . Between adjacent numbers, there are eight graduations, each of which corresponds to a length change of $0.022 \mathrm{~mm}$. One complete revolution of the SP $(0-4.4$ on the scale or 36 graduations) results in a length variation of $0.8 \mathrm{~mm}$. A setting of 2.4 , for example, in subsequent reporting refers to four smaller graduations above scale number 2 . Using the special tools provided by Anima Nova, one can change the SP length without taking it out of the violin body. 


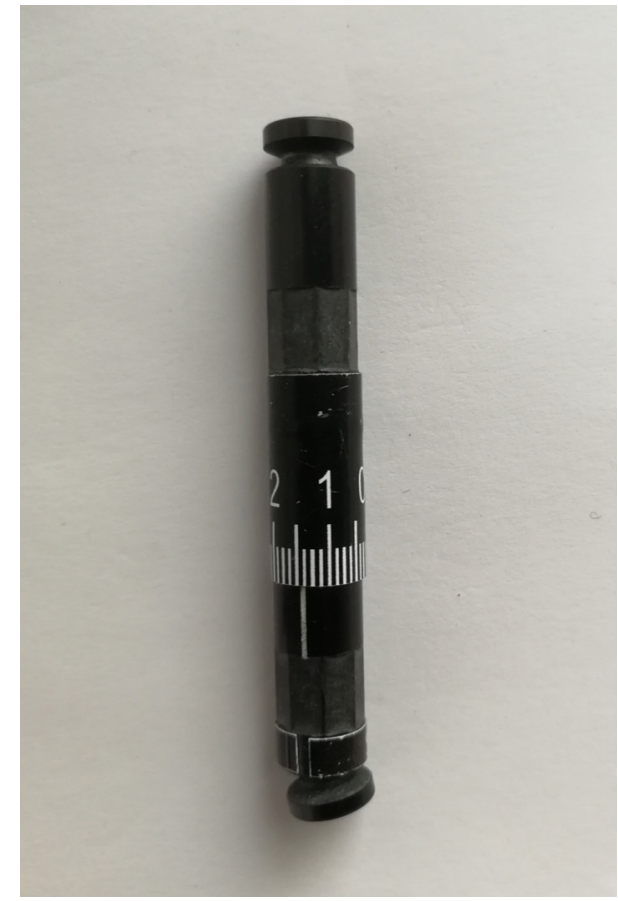

FIG. 1. (Color online) Anima Nova length-adjustable SP.

The violin used in this experiment is a performancelevel violin borrowed from Schulich School of Music, McGill University. We asked a local luthier to help replace the original wooden SP (around $53.77 \mathrm{~mm}$ high) with the Anima Nova length-adjustable SP, though subsequent adjustments were made by the experimenters. The lengthadjustable SP was placed about $3.5-4 \mathrm{~mm}$ below the bridge and centered with the treble foot of the bridge according to the SP manufacturer's instruction. The SP was set initially at the minimum length necessary to maintain its position, approximately $53 \mathrm{~mm}$.

\section{Participants}

Thirteen experienced violinists and six skilled luthiers participated in this experiment. Among the players, there were eight females, five males; seven native English speakers, three native Chinese speakers, and three other native speakers. Their average age was 30 years [standard deviation $(\mathrm{SD})=9$ years, range $=21-54$ years $]$. They had at least 16 years of playing experience $($ mean $=23$ years, $\mathrm{SD}=7$ years, range $=16-40$ years) and at least eight years of training (mean $=18$ years, $\mathrm{SD}=4$ years, range $=8-26$ years $)$. They reported playing $23 \mathrm{~h}$ per week on average $(\mathrm{SD}=10 \mathrm{~h}$, range $=6-37.5 \mathrm{~h}$ ). Eleven players described themselves as professional violinists. One of the players was a doctoral candidate in music performance, two had master's degrees in music performance, four were master students in music performance, three had bachelor's degrees in music performance, one had a bachelor's degree in arts, and two were currently undergraduate students in music. They reported playing various types of music. Among the luthiers, there were four males, two females; three native English speakers and three native French speakers. Their average age was 48.5 years $(\mathrm{SD}=11$ years, range $=36-61$ years $)$. They had at least 15 years of experience being a violin maker. Five luthiers played violin; among them there were one professional violinist, two advanced players, and two beginners. All subjects were paid for their participation.

\section{Detailed procedure}

This experiment consisted of two phases and lasted about $1 \mathrm{~h}$. Subjects were scheduled individually. Two experimenters were present during the experiment. One experimenter, who made adjustments to the SP, sat behind a table, with a screen in front to prevent subjects from observing the adjustments. The other experimenter helped with facilitating the experiment and taking notes for the subjects.

a. Phase 1: Optimal SP length. During the first phase, the SP was initially set at a length of $53 \mathrm{~mm}$ (the minimum necessary to hold its position). Subjects were then asked to play the violin with this initial setting and describe their feelings. Then the experimenter increased the SP length by 8 graduations (about $0.176 \mathrm{~mm}$ ), and the subjects repeated the playing and evaluation process. Subjects were informed that the experiment was about SP length modification using a length-adjustable SP before the experiment; however, they were not told in which direction the experimenter was adjusting the SP length. Subjects were asked whether the modification made the violin better or worse compared to the previous setting and to provide a verbal description of their perception of the change. If the subjects felt the setup was better or the same, the experimenter would continue to increase the SP length for a few graduations: 8 graduations or 4 graduations. If the subjects stated that the setup was worse, the experimenter would decrease the SP length for a few graduations: a decrease in 2-4 graduations to somewhere in between the two previous SP lengths. Then the subjects were asked to repeat the playing and evaluation procedure again. This process was repeated several times to find their most preferred length, with the number of graduations increased or decreased becoming smaller as the experiment continued. The whole process of searching for the most preferred length usually required 5-9 trials. During the SP length adjustment, the experimenter made sure that the SP length did not exceed $53.66 \mathrm{~mm}$ (30 graduations higher based on the original SP length of $53 \mathrm{~mm}$ ), beyond which it was feared the violin might be damaged. Each SP length adjustment took about a minute or less to complete. There was a 5-min break between phase 1 and phase 2 .

\section{b. Phase 2: Soundpost length difference} threshold. During the second phase, the experimenter randomly increased, decreased. or did not change the SP length in ten trials within a range of approximately $\pm 0.11 \mathrm{~mm}( \pm 5$ graduations) around each violinist's optimal length. In this way, the biggest length difference being tested was $0.11 \mathrm{~mm}$ ( 5 graduations). This range of length differences was chosen 
based on several pilot studies and feedback from luthiers and was expected to be beyond the perceptual threshold for length changes. The procedures used in the pilot studies were similar to those used for the listening test recordings (see Sec. III A 2 for details). Subjects were asked to play the violin during each trial and compare it with the previous setting, to decide whether they were the same or different. At the beginning of phase 2, subjects were asked to play the violin with their optimal SP lengths again. Then the experimenter increased, decreased, or did not change the SP length by different graduations over ten trials according to a plan determined in advance, which was unknown to subjects. The length variations were $\Delta L=0,0,2,-2,3,-3,4,-4,5,-5$ graduations (actual length of $\Delta L=0,0,0.044,-0.044,0.066$, $-0.066,0.088,-0.088,0.11,-0.11 \mathrm{~mm})$. They were randomized differently for each subject, while keeping the variations within $\pm 0.11 \mathrm{~mm}$ around the subjects' optimal length. To minimize subject fatigue, there was a 5-min break after five trials.

There were fewer "same" trials $(\Delta L=0)$ than "different" trials $(\Delta L \neq 0)$, though the number of "same" trials was equal to the number of "different" trials for a given non-zero $|\Delta L|$ (see Sec. II B 2). This was decided based on the detection theory model that was used to analyze the data (see the Appendix). Just noticeable differences are normally obtained by comparing a series of stimuli to the same reference stimulus. In this experiment, it would have been too tedious and tiring to always come back to the optimal SP length. The differences being small, we decided it was more practical to modify the length within a small range $( \pm 5$ graduations) around the optimal length and ask players whether there was a difference between this new length and the previous one. This constrained to some extent the order of the length differences (which was therefore only pseudorandom): for instance, a difference of +4 graduations could not follow a difference of +2 graduations, as this would have led to a length that was too far from the optimal length.

\section{B. Results}

\section{Optimal SP lengths}

During the first phase of the experiment, we found an optimal SP length for each subject. The optimal SP lengths of all subjects are represented relative to the original SP length (around $53 \mathrm{~mm})$ in Fig. 2, sorted from smallest $(0.132 \mathrm{~mm})$ to largest $(0.616 \mathrm{~mm})$. The minimum and maximum SP length variations that subjects evaluated were 0 and $0.66 \mathrm{~mm}$ relative to the original SP length $(53 \mathrm{~mm})$, respectively. The interquartile ranges of the relative optimal SP length for all subjects, players, and makers were, respectively, 0.33, 0.352, and $0.2805 \mathrm{~mm}$. The interquartile range for makers was smaller than for players, meaning that the optimal SP lengths for makers were more concentrated, which is also seen in Fig. 2. This could be due to the small number of maker participants. The median relative optimal SP length for makers $(0.308 \mathrm{~mm})$ was lower than for players $(0.396 \mathrm{~mm})$.

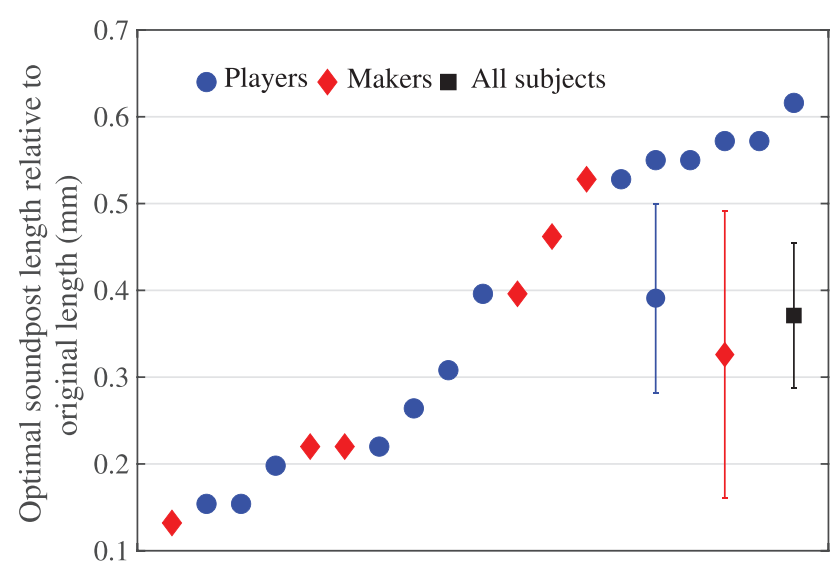

FIG. 2. (Color online) Optimal SP length relative to original length for every subject sorted from smallest to largest and mean optimal SP length relative to original length for all subjects, players, and makers (error bar $=95 \%$ confidence interval of the mean).

Figure 2 also displays the mean optimal SP length relative to the original SP length for all subjects, players, and makers separately. Error bars of the two-sided 95\% confidence interval of the means are also displayed. The mean relative optimal SP length and SD for all subjects were 0.371 and $0.171 \mathrm{~mm}$. The corresponding mean and SD for players and makers were 0.391 and $0.18 \mathrm{~mm}$ and 0.326 and $0.158 \mathrm{~mm}$, respectively. Players had a higher mean relative optimal SP length than makers. The confidence interval error bar of the means for makers was very large, which again might be partially due to the small number of maker participants. We compared the relative optimal SP length for players and makers by performing the independentsamples Mann-Whitney $U$ test (not employing independentsamples $t$ test for the violation of normal distribution assumption). The results showed that the null hypothesis that the distribution of the relative SP length was the same across players and makers could not be rejected, $U=28$, $z=-0.969, p=0.368$.

In addition to the perceptual experiment, we also conducted bridge admittance measurements for each SP length that had been evaluated while finding the optimal SP length for each subject. The results will be presented in a subsequent publication under development.

\section{Perceptual threshold of SP length differences}

As explained in the Appendix, the different responses to the two classes of stimuli can be classified in four categories, which are provided in Table I. Based on this

TABLE I. Different responses for different stimulus classes for the playing test.

\begin{tabular}{|c|c|c|}
\hline \multirow[b]{2}{*}{ Stimulus class } & \multicolumn{2}{|c|}{ Response } \\
\hline & "Different" & "Same" \\
\hline Different SP lengths $\left(S_{2}\right)$ & Hits & Misses \\
\hline Same SP length $\left(S_{I}\right)$ & False alarms & Correct rejections \\
\hline
\end{tabular}


classification, we can estimate the threshold of the SP length differences by calculating a sensitivity measure $d^{\prime}$ for each $\Delta L$ (see Appendix for details). During phase 2 of this experiment, positive $\Delta L$ and negative $\Delta L$ were counterbalanced by randomizing the presentation of positive $\Delta L$ and corresponding negative $\Delta L$ for subjects. In addition, to increase the number of test trials (sample size) and estimate the threshold more precisely, we calculated $d^{\prime}$ for each $|\Delta L|$ instead of each $\Delta L$. Thus, the number of trials for stimulus class $S_{1}$ was equal to the number of trials for each case of stimulus class $S_{2}$, as there were two zero-length variations among the ten length variations during phase 2 for each subject.

Figure 3(a) shows the probabilities that subjects considered the two SP lengths with a length difference of $|\Delta L|$ as "different." We can see that the false alarm rate, which corresponds to $P$ (“different") for $\Delta L=0$ was very high: 0.71 . It was even higher than the hit rate for $|\Delta L|=3$ graduations: 0.68. The highest hit rate was for $|\Delta L|=4$ graduations:

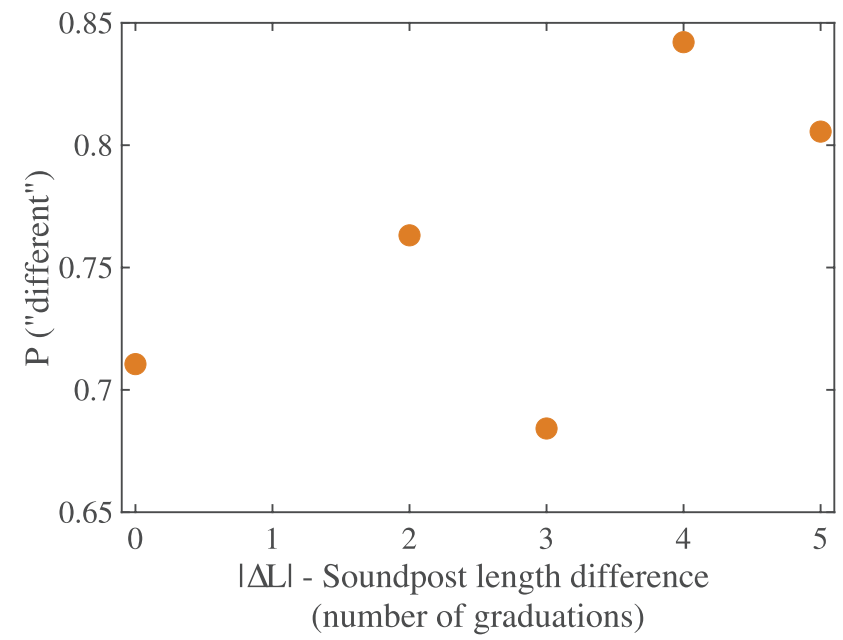

(a)

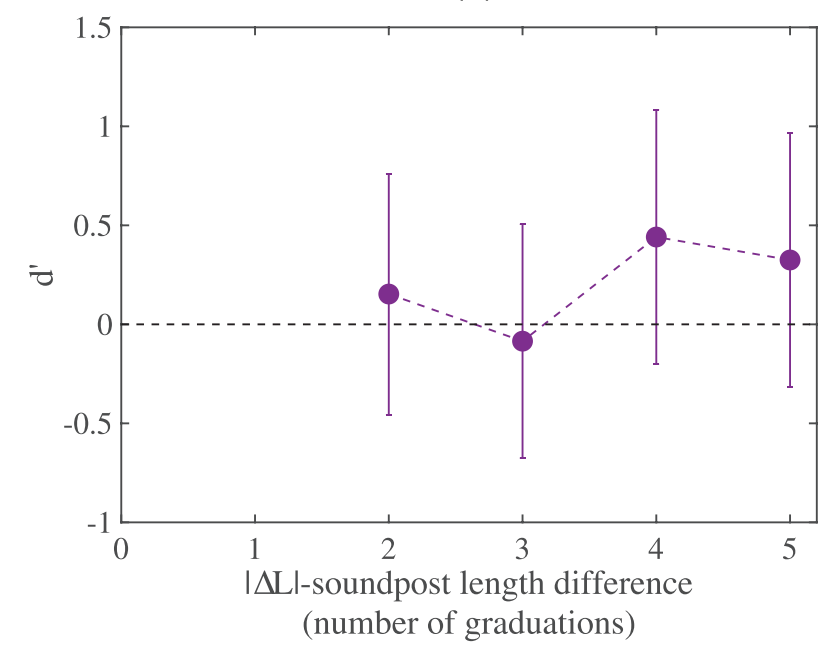

(b)

FIG. 3. (Color online) (a) Probabilities subjects considered the two SP lengths with a length difference of $|\Delta L|$ as "different"; (b) perceptual sensitivity $d^{\prime}$ for each $|\Delta L|$ (error bar $=95 \%$ confidence interval around $\left.d^{\prime}\right)$.
0.84. Players had a slightly lower false alarm rate (0.69) than makers (0.75). The $d^{\prime}$ for each $|\Delta L|$ is shown in Fig. 3(b). Error bars of $95 \%$ confidence interval around the $d^{\prime}$ are also displayed. We can see that the lower limits of the confidence intervals for all $|\Delta L|$ were smaller than 0 , implying that subjects could not recognize the SP length change of $0.11 \mathrm{~mm}$ ( 5 graduations) or less at better than chance levels. When analyzed separately for players and makers, the results were similar: neither players nor makers could recognize the SP length variation of $0.11 \mathrm{~mm}$ or less at better than chance levels.

\section{Discussion}

In this experiment, we explored violinists' and luthiers' perception of violin SP length differences through a playing test. By employing a length-adjustable SP, we were able to find the optimal SP length for each subject and investigate the perceptual sensitivity to SP length differences around each subject's optimal SP length.

The results showed that the subjects' optimal SP lengths varied from 0.132 to $0.616 \mathrm{~mm}$ relative to the original SP length $(53 \mathrm{~mm})$, reasonably well inside the extreme SP lengths that were tested $(0$ and $0.66 \mathrm{~mm})$. This shows that perceived violin quality first increased as the SP length increased and started to decrease after a certain length range, which is a phenomenon well known by makers. Also, the optimal SP lengths for subjects varied within an interquartile range of $0.33 \mathrm{~mm}$. The interquartile range was bigger for players $(0.352 \mathrm{~mm})$ than for makers $(0.2805 \mathrm{~mm})$. The mean optimal SP length relative to the original SP length (about $53 \mathrm{~mm}$ ) was also higher for players $(0.391 \mathrm{~mm})$ than for makers $(0.326 \mathrm{~mm})$. Statistical analysis showed that the differences of the relative optimal SP length for players and makers were not significant. Indeed, the fact that there was such a large range of optimal lengths showed that subjects did not agree on a "best" setting (even when only one parameter was changed). However, this range has to be interpreted with respect to the detection thresholds of players and makers. During the second phase of the experiment, the perceptual threshold of the SP length differences around each subject's optimal SP length was estimated by calculating a perceptual sensitivity measure of $d^{\prime}$. The results for all subjects showed that subjects could not recognize length changes of $0.11 \mathrm{~mm}$ or less at better than chance level.

Overall, the false alarm rate in evaluating the SP length differences was very high, i.e., subjects tended to say "different" even though there was no change at all in the SP length. That might partly be due to the sequential nature of the trials (they could not compare the different settings at the same time, and thus they might forget what the previous setting was like, though it only took a minute or less to make the SP changes). As well, there was a significant amount of variation in their approach to violin evaluation. Some subjects used a very consistent set of playing materials for each trial, while others used either very limited or changing materials between trials. Additionally, the 


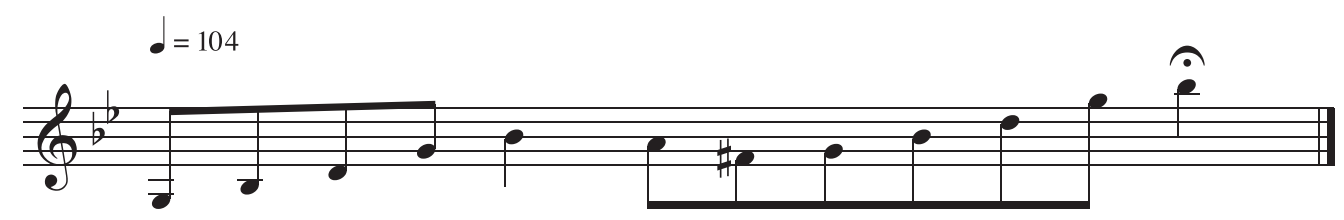

FIG. 4. Notated violin excerpt used for recording.

variation of SP length was quite small $(\leq 0.11 \mathrm{~mm})$, which made the task very difficult and could have contributed to player fatigue. The results indicate that the true perceptual threshold is beyond that range, even though initial pilot studies and discussions with makers seemed to suggest differently. Finally, there was some imprecision in SP adjustments, with an absolute average error of $0.007 \mathrm{~mm}$. All these factors could have had an effect on our results.

A playing test was initially chosen because it offers the advantage that participants could explore the violin with different SP lengths by themselves and would thus be able to evaluate the violin sound quality as well as playability. They could play freely in different registers of the violin and try various playing techniques to assess the violin's response. However, the disadvantage is that participants could not compare two different SP length settings in quick succession, and thus they might have forgotten what the previous setting was like. Moreover, variations in the way they played for the different length settings could also influence their judgment. For these reasons, we decided to conduct a listening test using recordings made on a violin with different SP lengths to further examine the perceptual threshold of violin SP length differences. This also afforded the opportunity to test a greater range of SP length differences.

\section{LISTENING EXPERIMENT}

\section{A. Materials and methods}

\section{General design}

The same length-adjustable carbon fiber SP used in the playing test was employed in this experiment. We recorded a soloist playing the same musical excerpt on a violin with different SP lengths. Violinists and luthiers were invited to listen to the recordings and provide perceptual feedback using a computer interface. Tasks included "same" or "different" pairwise comparisons.

\section{Recordings}

Recordings were made in an acoustically dry room to avoid having reverberant effects mask the violin sound. The surface area of the room was approximately $20 \mathrm{~m}^{2}$. A Sony (Tokyo, Japan) PCM-D100 was used for the recording. The recorder was placed on a 1-m high table facing the violinist. The table was approximately $1.5 \mathrm{~m}$ away from the violinist. The recordings were saved in 16-bit WAV format. The recording position was chosen to produce a natural quality of sound.
The violin used for the recordings was not the same as the one used in the previously described playing experiment. Before the recording, a violin maker helped replace the original wooden SP (around $54.88 \mathrm{~mm}$ high) with the Anima Nova length-adjustable SP. The length-adjustable SP was placed about $3.5 \mathrm{~mm}$ below the bridge and $2 \mathrm{~mm}$ inside the right end of the treble bridge foot according to the SP manufacturer's instructions. It was set initially at a relatively low length (around $54.11 \mathrm{~mm}$, reading on SP was 2.0). A professional violinist was invited to help make the recordings. The recorded violin was made and provided by a luthier, who was also present to adjust the length of the SP. The recording excerpt was based on the Max Bruch violin concerto and was an "abridged" version specifically for the listening test (see Fig. 4). The particular excerpt was chosen by the player because it incorporated the main register of the violin.

During the recording, the SP length was first increased in increments of 3 graduations $(0.066 \mathrm{~mm})$ over four iterations, up to a setting (reading 3.4 equivalent to $54.374 \mathrm{~mm}$ ) that was considered "tight" (see details in Table II). ${ }^{1}$ Then the SP was lowered to a reading of $2.2(54.154 \mathrm{~mm})$, below the length that the player preferred in the first procedure $(2.3$ or $54.176 \mathrm{~mm}$ ), and subsequently increased by increments of 1 graduation $(0.022 \mathrm{~mm})$ up to a setting of $2.5(54.220 \mathrm{~mm})$. Larger increments were used during the first SP length increasing procedure to search for the range that was considered "good," and smaller increments were used during the second SP length increasing procedure to find the closest "best" SP length. The player's most preferred SP length was 2.4 or $54.198 \mathrm{~mm}$ during the second SP length increasing procedure.

TABLE II. Recording process and recorded SP lengths (represented by the reading on SP, sorted by SP length); stimuli that were chosen for subsequent pairwise comparisons are designated by a recording number, which is shown in parentheses after the corresponding SP length, and they are available for listening in the supplemental material (see footnote 1).

\begin{tabular}{lcc}
\hline \hline \multicolumn{2}{l}{ Soundpost length increasing procedure } & \\
\hline 1 st & 2nd & Actual length (mm) \\
\hline $2.0(\mathrm{R} 1)$ & $2.2(\mathrm{R} 2 ; \mathrm{R} 3)^{\mathrm{a}}$ & 54.110 \\
& $2.3(\mathrm{R} 5)$ & 54.154 \\
$2.3(\mathrm{R} 4)$ & $2.4(\mathrm{R} 6)$ & 54.176 \\
& 2.5 & 54.198 \\
$2.6(\mathrm{R} 7)$ & & 54.220 \\
$3.1(\mathrm{R} 8)$ & & 54.242 \\
$3.4(\mathrm{R} 9)$ & & 54.308 \\
\hline
\end{tabular}

${ }^{\text {a Two different recordings on SP length } 2.2 \text { were chosen as two different }}$ stimuli. 


\section{Participants}

Thirteen experienced violinists and eight skilled luthiers participated in this experiment. Twelve of the subjects had participated in the playing test reported in Sec. II.

Among the players, there were eight females, five males; four native English speakers, four native Chinese speakers, three native French speakers, one native English and French speaker, and one native Italian speaker. Their average age was 32 years $(\mathrm{SD}=9.7$ years, range $=22-54$ years). They had at least 16 years of playing experience (mean $=26$ years, $\mathrm{SD}=8.8$ years, range $=16-40$ years $)$ and at least eight years of training (mean $=18$ years, SD $=4.6$ years, range $=8-23$ years). They reported playing $27 \mathrm{~h}$ per week on average $(S D=12 \mathrm{~h}$, range $=5-50 \mathrm{~h})$. Eleven players described themselves as professional violinists. One player had a soloist diploma, one had a doctoral degree, two were doctoral students in music performance, three had master's degrees in music performance, one was a master student in music performance, two had bachelor's degrees in music performance, one had a bachelor's degree in arts, and two were currently 4th-year undergraduate students in music performance. They reported playing various types of music.

Among the luthiers, there were six males, two females; four native English speakers, four native French speakers. Their average age was 45.7 years $(\mathrm{SD}=13$, range $=29-61)$. They had at least 10 years of experience as a violin maker (mean $=23.7, \mathrm{SD}=11$, range $=10-40$ ). Six luthiers played the violin, and among them there were one professional violinist, three advanced players, and two beginners. All subjects were paid for their participation.

\section{Stimuli}

The stimuli were created based on each recording made at each SP length. The mean stimulus exposure duration was around $4.66 \mathrm{~s}$. They were presented on a laptop in a relatively quiet environment using Sennheiser (Wedemark, Germany) HD 280 pro headphones and an external Apogee Duet (Santa Monica, USA) audio interface.

\section{Detailed procedure}

We selected nine recordings as stimuli, shown in Table I, eliminating the recordings with more obvious unintended playing artifacts in order not to affect the comparison.

Ten pairs were then chosen for comparisons as displayed in Table III. P1 and P2 were two pairs of identical recordings. P3 and P4 were two pairs of different recordings of the same SP lengths. We can see that the two recordings in P3 were made during the same SP increasing procedure, while the two recordings in $\mathrm{P} 4$ were made during the two different SP increasing processes (see Table II and Sec. III A 2 for details of the recording process). There were two tests for each subject with a 5-min break in between. Subjects listened to eight pairs of stimuli in each test. Pairs P5-P10 existed in both tests with stimuli order in each pair switched for the two tests. P1 and $\mathrm{P} 2$ existed in two different tests separately and randomly,
TABLE III. Pairs of stimuli used in the listening test.

\begin{tabular}{lccc}
\hline \hline Pair & $\begin{array}{c}\text { Length difference } \\
\text { (No. of graduations) }\end{array}$ & $\begin{array}{c}\text { Stimulus number and the corresponding } \\
\text { SP length with recording procedure }\end{array}$ \\
\hline P1 & 0 & R6 $(2.4,2 \mathrm{nd})$ & $\mathrm{R} 6(2.4,2 \mathrm{nd})$ \\
P2 & 0 & $\mathrm{R} 7(2.6)$ & $\mathrm{R} 7(2.6)$ \\
P3 & 0 & $\mathrm{R} 2(2.2,2 \mathrm{nd})$ & $\mathrm{R} 3(2.2,2 \mathrm{nd})$ \\
P4 & 0 & $\mathrm{R} 4(2.3)$ & $\mathrm{R} 5(2.3,2 \mathrm{nd})$ \\
P5 & 2 & $\mathrm{R} 3(2.2,2 \mathrm{nd})$ & $\mathrm{R} 6(2.4,2 \mathrm{nd})$ \\
P6 & 3 & $\mathrm{R} 1(2.0)$ & $\mathrm{R} 4(2.3)$ \\
P7 & 4 & $\mathrm{R} 1(2.0)$ & $\mathrm{R} 6(2.4,2 \mathrm{nd})$ \\
P8 & 6 & $\mathrm{R} 4(2.3)$ & $\mathrm{R} 8(3.1)$ \\
P9 & 9 & $\mathrm{R} 4(2.3)$ & $\mathrm{R} 9(3.4)$ \\
P10 & 12 & $\mathrm{R} 1(2.0)$ & $\mathrm{R} 9(3.4)$ \\
\hline \hline
\end{tabular}

aStimuli come from the first recording procedure unless specified as "2nd."

and this was the same case for $\mathrm{P} 3$ and $\mathrm{P} 4$. The presentation order of the pairs in each test and the stimuli in each pair were randomized for each subject.

Subjects were given instructions before the listening test as follows:

In this listening test, you will be presented with two tests; each consists of eight pairs of recordings.

Each pair of recordings will be played twice in the order of $\boldsymbol{A}-\boldsymbol{B}-\boldsymbol{A}-\boldsymbol{B}$, and you will only be able to click each pair a total of two times.

Please indicate whether you think the violin setup in each of the two recordings is the same setup or a different setup. If you think it is different, please describe the differences.

These recordings were made by one player on the same violin. Different adjustments were made to the violin between recordings, and the player was asked to perform the excerpt as similarly as possible each time, but there still may be slight changes in his playing (tempo, articulation, dampening of the strings...). Please ignore the differences in his playing and decide whether the setup is the same or different.

Subjects who had participated in the playing test were aware that the "setup" indicated in the instructions meant SP length. For consistency, we told subjects who did not take part in the playing test that "different setup" meant "different SP length" and showed the Anima Nova SP leaflet to them and explained the SP working principle.

Before the formal listening test, we played all the stimuli to the subjects so that they could get an initial impression of the recordings. The differences were quite subtle and difficult to hear according to several pilot studies we had previously conducted. This was also the reason we put two trials together (A-B-A-B), as two iterations of each pair seemed necessary. The formal listening test of pair comparisons of the stimuli was then performed through a computer interface created in матцAв. Further details about the test process through the interface are available in $\mathrm{Fu}(2020)$.

The perceptual sensitivity was estimated with the same method as in the playing experiment. However, in this case, 
TABLE IV. Different responses for different stimulus classes for the listening test.

\begin{tabular}{|c|c|c|}
\hline \multirow[b]{2}{*}{ Stimulus class } & \multicolumn{2}{|c|}{ Response } \\
\hline & "Different" & "Same" \\
\hline $\begin{array}{l}\text { Different SP length } \\
\text { recordings }\left(S_{2}\right)\end{array}$ & Hits & Misses \\
\hline Identical recording $\left(S_{I a}\right)$ & False alarms & Correct rejections \\
\hline $\begin{array}{l}\text { Different recordings of the } \\
\text { same SP length }\left(S_{1 b}\right)\end{array}$ & False alarms & Correct rejections \\
\hline
\end{tabular}

there were two different types of reference stimulus classes $\left(S_{I a}\right.$ and $\left.S_{I b}\right)$, as shown in Table IV, and for each type of the reference stimulus class, we employed two reference pairs, which were shown in Table III. An additional type of reference stimulus class, $S_{1 b}$ ("different recordings of the same SP length"), was added to estimate the effects of possible slight variations in playing technique. That is, even though subjects were told that they should ignore variations in playing technique, there was still the possibility that slight playing variations could be interpreted as different SP lengths. The $S_{1 a}$ class consisted of pairs $\mathrm{P} 1$ and $\mathrm{P} 2$, while $S_{1 b}$ included P3 and P4. The reason why we selected two different pairs in the $S_{I a}$ class was for variety, while the reason for $S_{1 b}$ was to illustrate various differences that could be caused by playing technique variations.

\section{B. Results}

As in the playing experiment, the threshold of the SP length differences in this experiment was estimated through the calculation of a sensitivity measure $d^{\prime}$ for each $|\Delta L|$. Figure 5 shows the probabilities (also known as hit rates) that subjects considered the two SP lengths with a difference of $2,3,4,6,9$, or 12 graduations as "different," respectively. We can see a trend that the hit rate increases as the SP length difference increases except that the hit rate for 3 graduations was extraordinarily higher than its adjacent intervals. Figure 5 also displays the probabilities (also known as false alarm rates) that subjects considered the first type of reference pairs $\left(S_{1 a}\right)$ that consisted of identical recordings (P1 [R6, R6] and P2 [R7, R7]) and the second type of reference pairs $\left(S_{1 b}\right)$ that consisted of different recordings of the same SP lengths (P3 [R2, R3] and P4 [R4, R5]) as "different," respectively. We can see that the false alarm rates of the reference pairs $S_{1 a}$ were much lower than the false alarm rates of the reference pairs $S_{1 b}$. Among the two reference pairs of $S_{l b}$, the false alarm rate of P4 [R4, R5] $(p=0.619)$ was much higher than P3 [R2, R3] $(p=0.381)$. Looking back to Table II, we can see that stimuli R2 and R3 were recorded during the same SP increasing process, while stimuli R4 and R5 were recorded at different phases of the SP length adjustment procedure and thus at significantly different times. Thus, it appears likely that variations in the timing of recordings and/or slight variations in

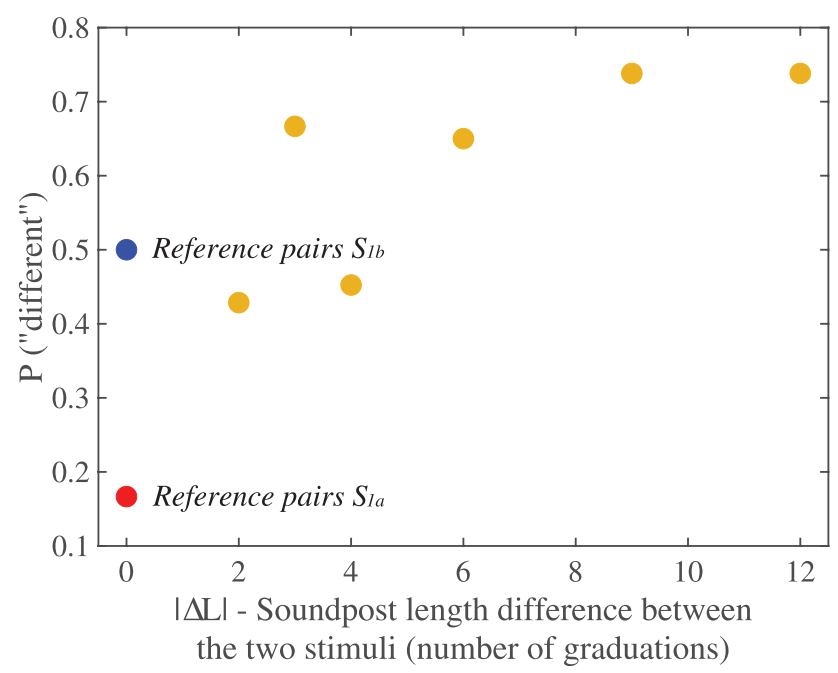

FIG. 5. (Color online) Probabilities that subjects considered each pair of stimuli as different, when the pairs consisted of recordings made at two different lengths or at the same length.

playing technique contributed to inaccuracies in the evaluation of SP length.

Figure 6 shows the perceptual sensitivity measure $d^{\prime}$ for each $|\Delta L|$ calculated based on the two types of reference pairs, respectively. In addition, we also calculated the perceptual sensitivity of reference pairs $S_{l b}$ (i.e., $|\Delta L|=0$ ) based on the reference pairs $S_{l a}$. Error bars of the two-sided 95\% confidence interval around $d^{\prime}$ are also displayed. We can see that all $d^{\prime}$ that were calculated based on the reference pairs $S_{l a}$ were greater than 0 , including the $d^{\prime}$ for the reference pairs $S_{I b}$. It implies that subjects could differentiate all different stimuli we presented at above chance levels. However, we could not conclude that they could recognize all SP length differences because they did not manage to distinguish the differences caused by SP length from those

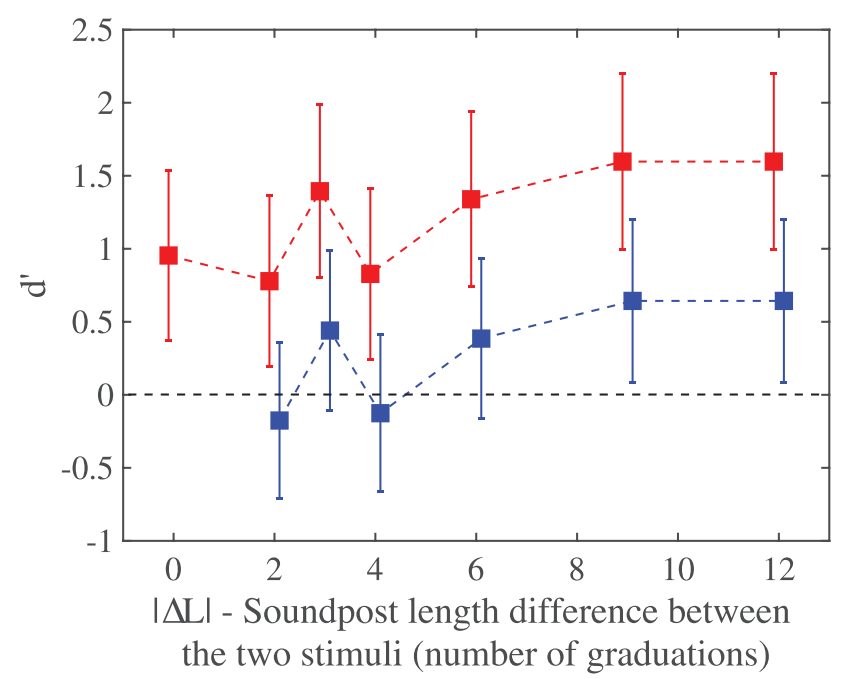

FIG. 6. (Color online) Perceptual sensitivity $d^{\prime}$ for each $|\Delta L|$ calculated using two sets of references: the upper line represents the $d^{\prime}$ calculated based on reference pairs $S_{l a}$, and the lower line represents the $d^{\prime}$ calculated based on reference pairs $S_{1 b}$. 
by playing technique. For $d^{\prime}$ that was calculated based on the reference pairs $S_{l b}$, we can see that only $d^{\prime}$ values for SP length differences of 9 and 12 graduations were a bit greater than 0 . This means that subjects could only differentiate different SP lengths with a difference of 0.198 and $0.264 \mathrm{~mm}$ at above chance level based on the reference pairs $S_{l b}$.

During the listening test, subjects were asked to describe the differences they heard if they thought the violin setup in each of the two recordings was different. By examining the verbal descriptions provided by the subjects, we found that subjects were generally consistent across the two tests in determining whether the two stimuli in each pair were the same or different as well as in describing the differences between them. Also, listeners in general agreed with the player's opinion during the recording, i.e., subjects agreed that the violin sound quality increased as the SP length increased up to a point where it then began to deteriorate. For instance, stimulus R6 (2.4, 2nd) in P5, stimulus R4 (2.3) in P6, and stimulus R4 (2.3) in P9 had more positive comments and fewer negative comments compared to stimulus R3 (2.2, 2nd), stimulus R1 (2.0), and stimulus R9 (3.4) in the respective pair. Neither stimulus R1 (2.0) nor stimulus R9 (3.4) in P10 had more positive or negative comments than the other, which is also in agreement with an optimal length that is between the two extremes. Only P7 and P8 had slightly contradictory results to this general tendency. Those two pairs included one recording that was among the player's most preferred recordings (one in each recording procedure); however, the difference between the number of positive and negative comments about the two recordings in any of the two pairs was not that large. For the reference pairs $S_{l b}$ of P3 and P4, we found that subjects showed preference toward one of the two recordings made at the same SP length, which again implies that listeners could not differentiate the differences caused by different SP lengths from those caused by variations of the player's technique. Also, for P4, the two recordings were made at the same SP length but in different recording procedures; the slight inaccuracy of the adjustable SP could also contribute to the differences between the two recordings.

\section{Discussion}

In this experiment, we explored players and makers' perception of different SP lengths through a listening test (using recorded sounds) with a computer interface. A violin installed with a length-adjustable SP was used to make the recordings. A concert violinist was invited to repeatedly perform a musical excerpt for different SP lengths. Subjects then compared six pairs of recordings at different SP lengths as well as two reference pairs of identical recordings and two reference pairs of different recordings at the same SP lengths.

The perceptual threshold of the SP length differences was estimated by calculating a perceptual sensitivity measure of $d^{\prime}$. The results showed that based on the two reference pairs $S_{1 a}$ of identical recordings, subjects could differentiate all different stimuli we presented at above chance levels, including the stimuli in the reference pairs $S_{l b}$ of different recordings made at the same SP lengths. However, based on the reference pairs $S_{1 b}$ of different recordings at the same SP lengths, subjects could only differentiate SP lengths with a difference of 9 or 12 graduations (i.e., 0.198 or $0.264 \mathrm{~mm}$ ) at above chance level.

Comparing the results of the listening test and the playing test in Sec. II, we found that the false alarm rates were much lower in the listening test than the playing test, with even the highest false alarm rate $(p=0.619)$ of the reference pair P4 [R4, R5] in the listening test lower than the false alarm rate $(p=0.711)$ in the playing test. The reasons may be that there were fewer variables in the listening test than in the playing test, which we have discussed in Sec. IIC, and that subjects could compare two stimuli in quick succession. In the listening test, we managed to test bigger SP length differences that we did not include in the playing test $(6,9$, and 12 graduations) and found that subjects were able to recognize SP length differences of 9 and 12 graduations at better than chance level in the listening test.

The verbal descriptions in the listening test revealed that listeners generally agreed with the player's opinions during the recording: the violin sound quality increased as the SP length increased up to a point where it then began to deteriorate.

Analyses of the reference pairs $S_{1 b}$ of different recordings at the same SP lengths imply that listeners could not differentiate the differences caused by different SP lengths from those caused by inadvertent variations of the player's technique. This is the primary disadvantage of the listening test.

\section{CONCLUSIONS}

In this study, we explored the perceptual influences of the SP length on the violin from the perspective of players and luthiers through a playing test and a listening test. A length-adjustable SP was employed for the experiments.

During the playing test, subjects were asked to play a violin with different SP lengths to find their optimal SP length, and then we explored subjects' perceptual sensitivity to SP length variations around each subject's optimal length. The results showed that subjects' optimal SP length varied from 0.132 to $0.616 \mathrm{~mm}$ relative to the original SP length $(53 \mathrm{~mm})$. Such a large range of optimal lengths implies a lack of agreement among players for a "best" setting, even when only the SP length is changed. This finding is consistent with a previous study (Saitis, 2012): a large amount of inter-individual variability existed in the preference rankings of violins. During the second phase of the playing experiment, we found that subjects could not recognize length changes of $0.11 \mathrm{~mm}$ or less at better than chance levels.

The true perceptual threshold of violin SP length variations may be difficult to find through a playing experiment because of the existence of a large number of variables and the sequential presentation of the trials. For these reasons, 
we conducted a listening test (using recorded sounds made by a soloist) investigating subjects' perceptual threshold as well. The results showed that subjects could differentiate SP lengths with a difference of 0.198 or $0.264 \mathrm{~mm}$ at a bit above chance level based on the reference pairs of different recordings at the same SP lengths. The false alarm rate in the listening test was much lower than in the playing test, which could be explained by the fact that the listening test had fewer variables than the playing test. The main disadvantage of the listening test was that listeners could not distinguish the differences caused by different SP lengths from those caused by inadvertent variations of the player's technique.

The playing experiment provided an experience similar to that of a violinist evaluating adjustments of an instrument when at a luthier shop. The length differences that were tested by subjects were decided based on several pilot studies and discussions with makers. However, the results indicate that the range of length variations used in the playing test were below the true perceptual threshold. Also, the fact that subjects tended to think a change had been made, even though they were told that changes would not always occur, demonstrates a certain bias in the mindset of players that they should be able to notice even very slight adjustments to their instruments. That said, in the listening test, we found that subjects could differentiate SP lengths with a difference of 0.198 and $0.264 \mathrm{~mm}$ at a bit above chance level based on the reference pairs of different recordings at the same SP lengths. These length differences are quite small, which supports the statements of luthiers that SP tightness is important. However, our findings do not seem to support their extreme belief that removing "just a shaving" from the SP can make a big difference.

The fact that small length differences can be detected will increase the difficulty of conducting an experiment to address another SP parameter, its position, which has certainly an equal or greater influence on the playing qualities. It is, however, extremely hard to control very precisely the position, and a small change in position will lead to a small change in tightness, which we now know can be perceived. It will thus be crucial to control both parameters independently.

More generally, this study shows once more that exploring luthiers' and players' beliefs in a scientific way is rather tricky. Designing a controlled experiment without "throwing the baby out with the bath water" is difficult. For instance, listening tests allow greater control but remove a large number of cues that are usually available to players when evaluating a violin and force the evaluation on just the sound quality. In addition, a lot of trials are usually needed to derive robust and statistically significant conclusions, but this implies a long duration of the experiment that will cause fatigue and therefore make participants less sensitive and reliable. Finally, the inter-individual variability, in terms of perception, preference, expertise, and verbal descriptions, is always very large, making the interpretation of the results rather complex.

\section{ACKNOWLEDGMENTS}

This study was funded by the Centre for Interdisciplinary Research in Music Media and Technology (CIRMMT), the Natural Sciences and Engineering Research Council of Canada (NSERC), the Hector and Ada Ma China Scholarship Council Fellowship, and the China Scholarship Council (CSC). We would like to thank the Schulich School of Music for loaning the violin and Senior and Junior Pal Molnar for loaning the Anima Nova length-adjustable SP. We acknowledge the violinist Nathan Giem for making the recordings; the luthiers Melina Polizzi, Monica Fortin, Meike Aupperle, and Sam Zygmuntowicz, who helped with installing and adjusting the SP during the recording; and all subjects who participated in the experiments. We are grateful to luthier Tom Wilder for replacing the SP and for fruitful discussions before the experiment and luthier Paul Noulet and violinist Lea Menissier for pilot study recordings. Finally, we thank Stephen McAdams, Catherine Guastavino, Arthur Paté, and Xin Wang for their helpful suggestions.

\section{APPENDIX}

Thresholds were estimated using detection theory (Macmillan and Creelman, 2005). We have two stimulus classes: length variations of $\Delta L=0$ are class $S_{1}$, and $\Delta L=$ $2,-2,3,-3,4,-4,5,-5$ graduations are different cases of the $S_{2}$ class. A "hit" is defined as a correct identification of an $S_{2}$ class element (participants recognize a length change); failing to identify it is a "miss." A "false alarm" is defined as an incorrect identification of an $S_{1}$ class element (they thought the length changed when no variation of the SP length was made); correctly responding "same" is a "correct rejection." Table I summarizes the four possible cases. The hit rate $(H)$ is the proportion of different SP lengths $\left(S_{2}\right)$ to which the subject responds "different," and the false alarm rate $(F)$ is the proportion of the same SP length $\left(S_{1}\right)$ similarly (but incorrectly) assessed. The hit and false alarm rates can be written as the following conditional probabilities:

$$
\begin{aligned}
& H=P\left({ }^{\prime \prime} \text { different }^{\prime \prime} \mid S_{2}\right), \\
& F=P\left({ }^{\prime \prime} \text { different }^{\prime \prime} \mid S_{1}\right) .
\end{aligned}
$$

The perceptual sensitivity is estimated using the $d^{\prime}$ measure: $d^{\prime}$ is defined in terms of the inverse of the normal distribution function $z$

$$
d^{\prime}=z(H)-z(F) .
$$

The hit or false alarm rate was thus converted to a $z$ score (i.e., to SD units) by the $z$ transformation. The $z$ transformation converts a proportion of 0.5 into a $z$ score of 0 , larger proportions into positive $z$ scores, and smaller proportions into negative $z$ scores. Thus, when $H=F, d^{\prime}=0$, and the performance is at chance; when $H>F, d^{\prime}>0$, which means that subjects are able to recognize a difference in 
length. The sensitivity of detection increases as $d^{\prime}$ increases. By calculating $d^{\prime}$ for each $\Delta L$, we can estimate the sensitivity of the subjects in SP length variation.

The standard error of $d^{\prime}$ was calculated according to Gourevitch and Galanter (1967). The variance (square of the standard error) of $d^{\prime}$ is the sum of the variances of the two (independent) transformed proportions: $z(H)$ and $z(F)$. Gourevitch and Galanter showed that observed $z$ scores are approximately normally distributed, with variance

$$
\operatorname{var}[z(p)]=\frac{p(1-p)}{N[\varnothing(p)]^{2}}
$$

where $\varnothing(p)$ is the height of the normal density function at $z(p)$ and $N$ is the number of trials. Consequently,

$$
\operatorname{var}\left(d^{\prime}\right)=\frac{H(1-H)}{N_{2}[\varnothing(H)]^{2}}+\frac{F(1-F)}{N_{1}[\varnothing(F)]^{2}},
$$

where $N_{2}$ and $N_{l}$ are the number of trials in stimulus class $S_{2}$ and $S_{l}$, respectively.

Values of the function $\varnothing$ can be computed

$$
\varnothing(p)=\frac{2}{\sqrt{2 \pi}} e^{-\frac{1}{2} z(p)^{2}} .
$$

By extending 1.96 standard errors (arithmetic square root of the variance) above and below observed $d^{\prime}$, we can obtain a $95 \%$ confidence interval around $d^{\prime}$.

\footnotetext{
${ }^{1}$ See supplementary material at https://www.scitation.org/doi/suppl/ $10.1121 / 10.0005587$ to listen to the recordings that were used in the listening test.
}

Bissinger, G. (1995). "Some mechanical and acoustical consequences of the violin soundpost," J. Acoust. Soc. Am. 97(5), 3154-3164.

Fu, L. (2020). "Exploring the perception of violin qualities: Student- vs. performance-level instruments, strings and soundpost height," $\mathrm{Ph} . \mathrm{D}$. thesis, McGill University, Montreal, Canada.

Gough, C. (2017). "The influence of the bridge, island area, bassbar and soundpost on the acoustic modes of the violin," in Proceedings of 2017 International Symposium on Musical Acoustics (ISMA), June 18-22, Montreal, Canada, pp. 67-70.

Gough, C. (2018). "The violin bridge-island input filter," J. Acoust. Soc. Am. 143(1), 1-12.

Gourevitch, V., and Galanter, E. (1967). "A significance test for one parameter isosensitivity functions," Psychometrika 32(1), 25-33.

Jansson, E. V. (2004). “Acoustics for violin and guitar makers," Kungliga Tekniska Högskolan, Department of Speech, Music and Hearing, http:// www.speech.kth.se/music/acviguit4/ (Last viewed June 5, 2019).

Jansson, E., Molin, N. E., and Sundin, H. (1970). "Resonances of a violin body studied by hologram interferometry and acoustical methods," Phys. Scr. 2(6), 243-256.

Johnson, C., and Courtnall, R. (1999). The Art of Violin Making (Robert Hale, London, UK).

Macmillan, N. A., and Creelman, C. D. (2005). Detection Theory: A User's Guide (Lawrence Erlbaum Associates, Mahwah, NJ).

Nadarajah, M. C. (2018). "The mechanics of the soundpost in the violin," Ph.D. thesis, University of Cambridge, Cambridge, UK.

Saitis, C., Giordano, B. L., Fritz, C., and Scavone, G. P. (2012). "Perceptual evaluation of violins: A quantitative analysis of preference judgments by experienced players," J. Acoust. Soc. Am. 132(6), 4002-4012.

Saldner, H. O., Molin, N. E., and Jansson, E. V. (1996). "Vibration modes of the violin forced via the bridge and action of the soundpost," J. Acoust. Soc. Am. 100(2), 1168-1177.

Savart, F. (1840). “The violin,” L'Institut 8, 54-56 [English Translation by D. A. Fletcher and reprinted in Musical Acoustics, Part I: Violin Family Components, Vol. 5 of Benchmark Papers in Acoustics, edited by C. M. Hutchins (Dowden, Hutchinson and Ross, Stroudsburg, PA, 1975), pp. 323-327].

Schelleng, J. C. (1971). "The action of the soundpost," Catgut Acoust. Soc. Newsl. 16, 11-15. 